\title{
Physiochemical Effects of Nanoparticle on Cell Nuclear Complex Pore \\ Transport: A Coarse-Grained Computational Model
}

Liuyang Zhang ${ }^{\mathrm{a}^{*}}$, Matthew Becton ${ }^{\mathrm{b}}$, Ning Liu ${ }^{\mathrm{b}}$, Rodney D. Averett ${ }^{\mathrm{b}}$, Ramana Pidaparti ${ }^{\mathrm{b}}$, and Xianqiao Wang ${ }^{b^{*}}$

${ }^{a}$ State Key Laboratory for Manufacturing Systems Engineering, Xi'an Jiaotong University, Xi'an, Shaanxi 710049, China

${ }^{\mathrm{b}}$ College of Engineering, University of Georgia, Athens, GA 30602, USA

*Corresponding authors: liuyangzhang@xjtu.edu.cn; xqwang@uga.edu

\section{(1) Effect of rigidity of NE plane}

In order to demonstrate the effect of rigidity of NPC pore, we performed transport process simulations of inert spherical NP (radius $=15 \sigma$ ) through a rigid NPC pore and a flexible NPC pore respectively, and results are shown in Figure S1 and Figure S2. The deformation of the NPC indicates that the fulfillment of large cargo translocation needs to overcome a large energy barrier exerted from the NPC.

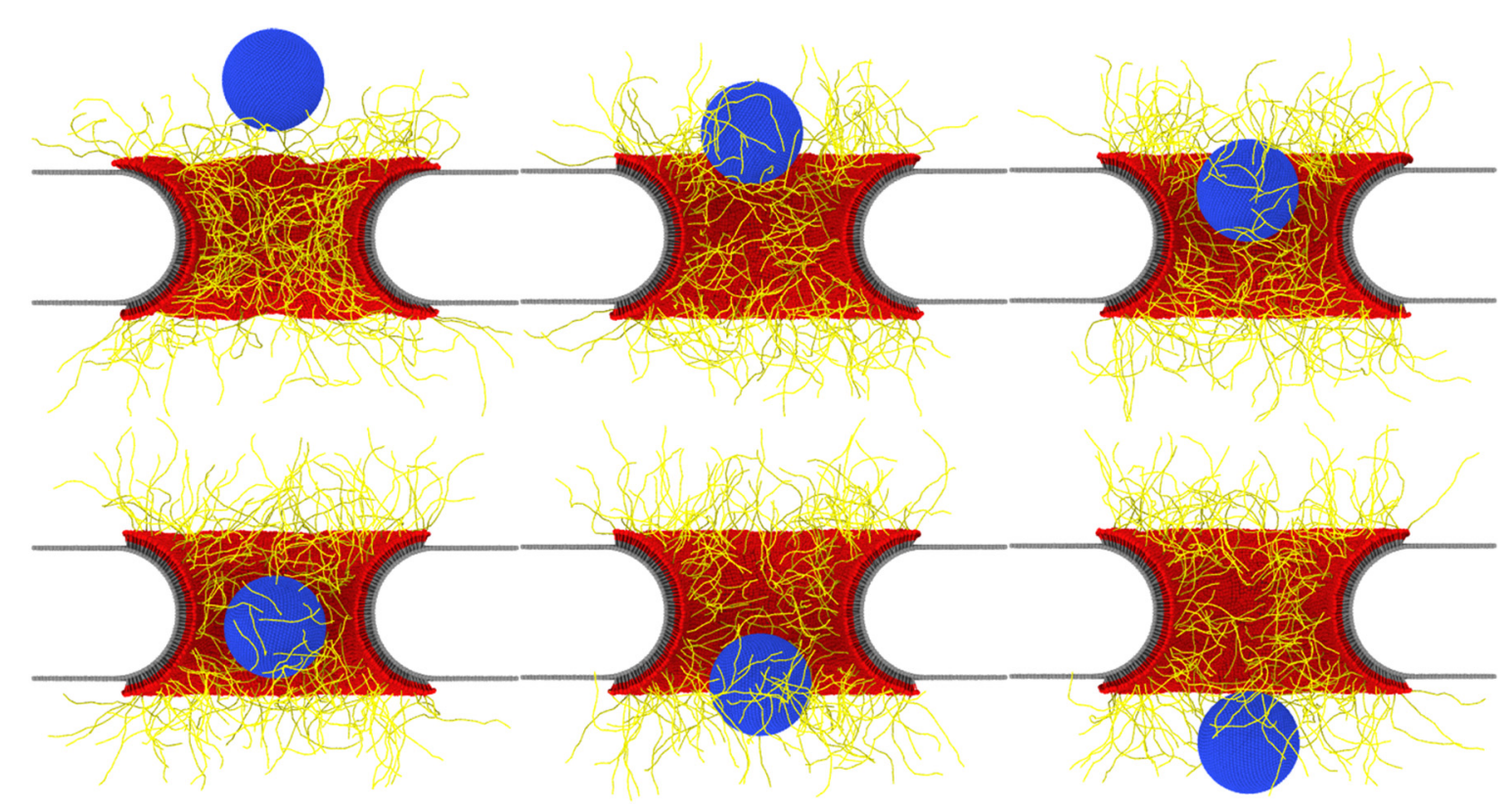

Figure S1. Dynamic inert spherical NP transport process through the NPC channel with rigid NE plane. 


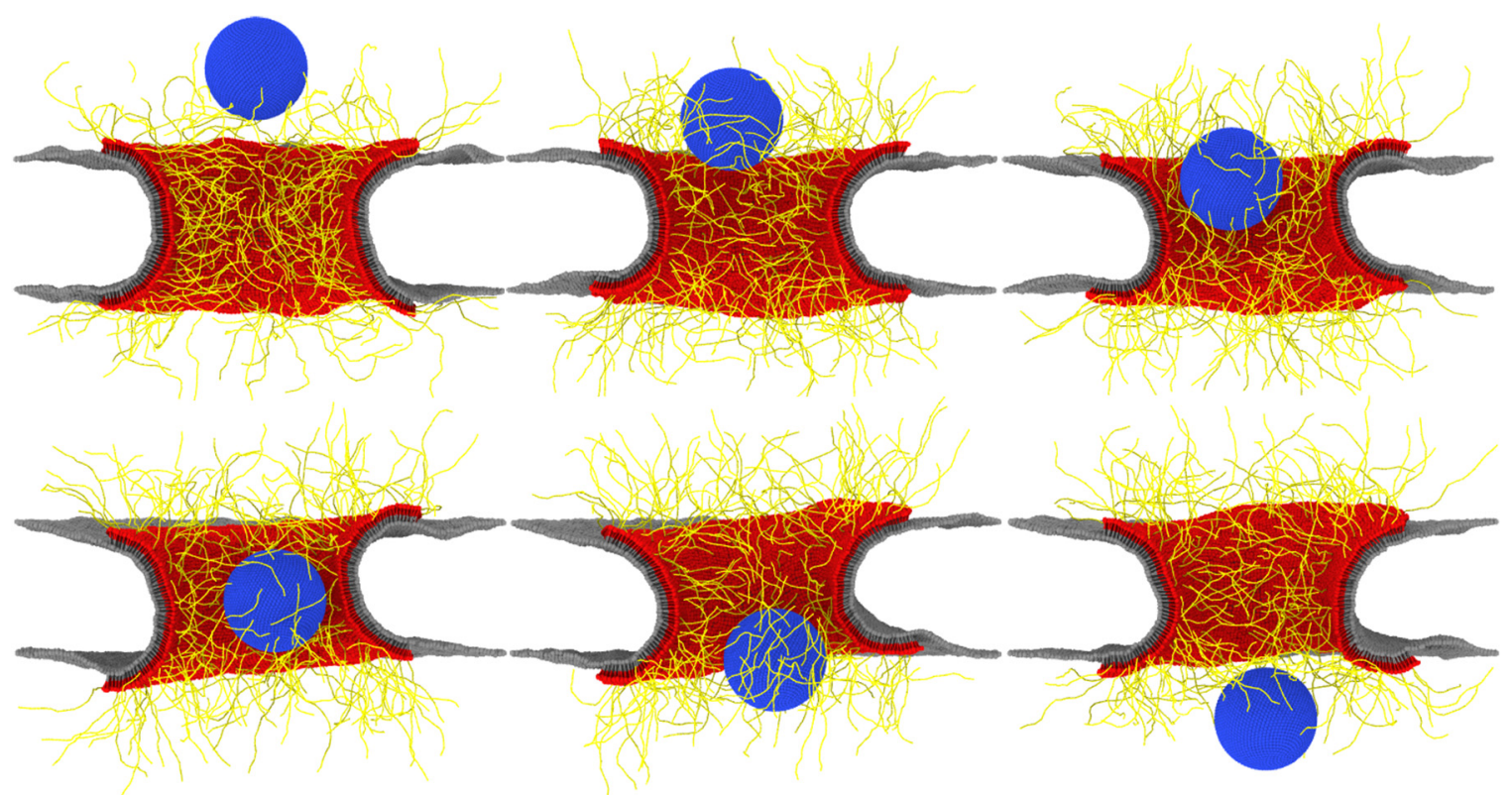

Figure S2. Dynamic inert spherical NP transport process through the NPC channel with flexible NE plane. From the evolution of free energy profile in Figure S6, it shows that the energy barrier for the NP transport through the flexible NPC pore is greater than that for the rigid NPC pore thanks to the energy consumption in the pore deformation

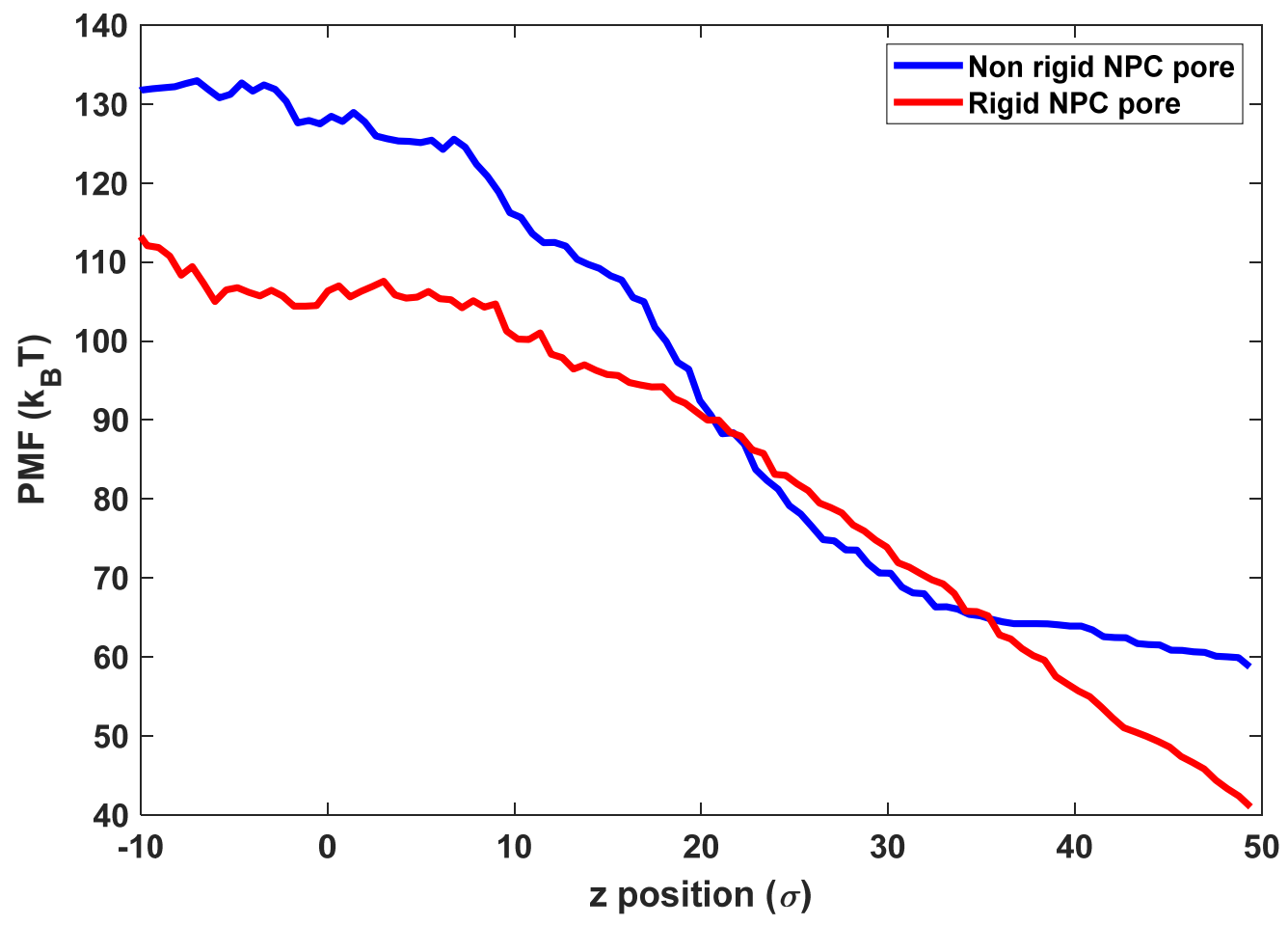

Figure S3. Effect of rigidity of NPC pore on the free energy profiles of spherical NP's transport process through the NPC channel. 


\section{(2) Free energy analysis}

In order to better understand the dynamic passive transport process, free energy analysis is used to quantitatively characterize the effect of a NP's size on its transport process via the calculation of the required energy penalty. The calculation of required free energy is based on the Steered Molecular Dynamic (SMD). SMD simulation is essentially Umbrella Sampling simulation if the velocity of pulling is very small. ${ }^{2}$ The conformational change in systems is induced by pulling the NP down towards the cell membrane with a constant pulling velocity of $0.025 \sigma / \tau{ }^{3}$ The potential of mean force (PMF) was computed based on Jarzynski's equality. ${ }^{4}$ A parameter $p(z)$ is used to measure the normal distance from the centre of a NP to the mid-plane of the NPC in the $\mathrm{z}$ direction as

$$
\mathrm{p}(\mathrm{z})=\mathrm{p}(\mathrm{z}=50)-\mathrm{z}[\mathrm{p}(\mathrm{z}=50)-\mathrm{p}(\mathrm{z}=0)]
$$

When $\mathrm{z}=50 \sigma$, it corresponds to the initial position of the NP above the NPC channel. As the NP pierces into the FGN domain inside the NPC channel, $\mathrm{z}$ gradually decreases and finally reaches to $\mathrm{z}=0 \sigma$ when the NP arrives at the mid-plane of the NPC. The free energy in this process is expressed as

$$
E=\int_{z=0}^{z=50} \frac{\partial E(z)}{\partial z} d z
$$

When the pulling velocity is small, the NP transport path can be discretised to a series of $\mathrm{z}$ values between $50 \sigma$ and $0 \sigma$. For each z position, a harmonic potential

$$
\mathrm{U}(\mathrm{z})=\frac{\mathrm{k}_{\mathrm{z}}}{2}[\mathrm{p}(\mathrm{z})-\mathrm{Z}]^{2}
$$

is imposed to confine the motion of the NP in the z-direction, where $\mathrm{k}_{\mathrm{z}}=100 \mathrm{k}_{\mathrm{B}} \mathrm{T} / \sigma$ and $\mathrm{p}(\mathrm{z})$ are the spring constant and equilibrium position of the potential, respectively; $\mathrm{Z}$ is the position of the NP center. Under the harmonic constraint, the NP is forced to oscillate around a pseudoequilibrium position $\langle Z\rangle$ in the vicinity of $\mathrm{p}(\mathrm{z})$, where $\langle Z\rangle$ is the ensemble-averaged position of the NP center. The derivative of the free energy is determined from the constrained interaction between the coated NP and its surrounding as

$$
\frac{\partial \mathrm{E}(\mathrm{z})}{\partial \mathrm{z}}=\left\langle\frac{\partial \mathrm{U}(\mathrm{z})}{\partial \mathrm{z}}\right\rangle=\mathrm{k}_{\mathrm{z}}[\mathrm{p}(\mathrm{z})-\langle\mathrm{Z}\rangle]
$$

By integrating this expression allows the free energy change during the whole transport process to be determined as a function of the distance from the bilayer midplane 


$$
E=\int_{p(z=0)}^{p(z)} k_{z}[p(z)-\langle Z\rangle] d z
$$
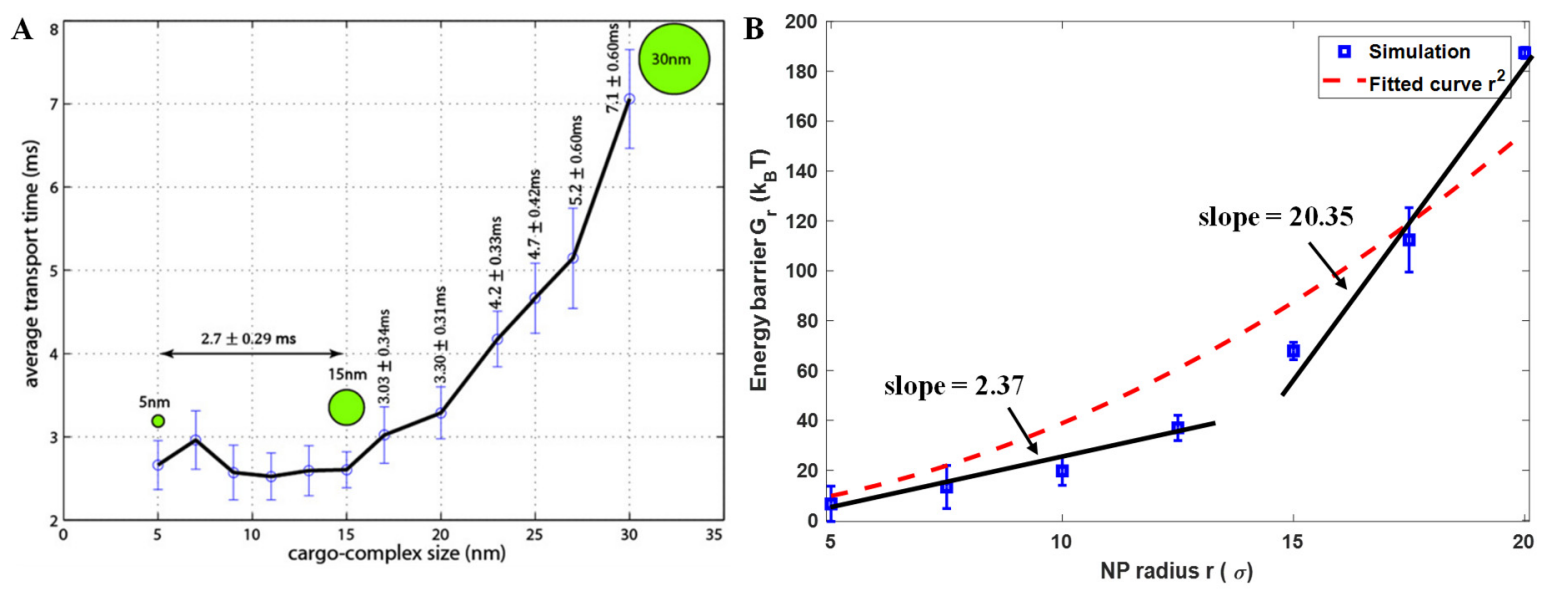

Figure S4. (A) Average transport time VS cargo size (5nm-35nm) from R. Moussavi-Baygi’s work. ${ }^{1}$; (B) energy barrier vs NP size (5nm-20nm)

\section{(3) Transport pathway for inert NP}

For the active NP, the main translocation path is dependent on the NP size, but mainly close to the central channel wall. For the inactive NP, the interaction between the NP and FGN is dominated as repulsive force. However, for the inactive NP, From Figure S5, it is clearly observed that the translocation path is close to the central axis. It can be attributed to that for all orientations, that the lowest energy pathway for an inert NP passing through the NPC channel is close to the central axis. The restriction of the position of the NP to the central axis of the channel can be rationalized via the contact between the NP and FGN. 


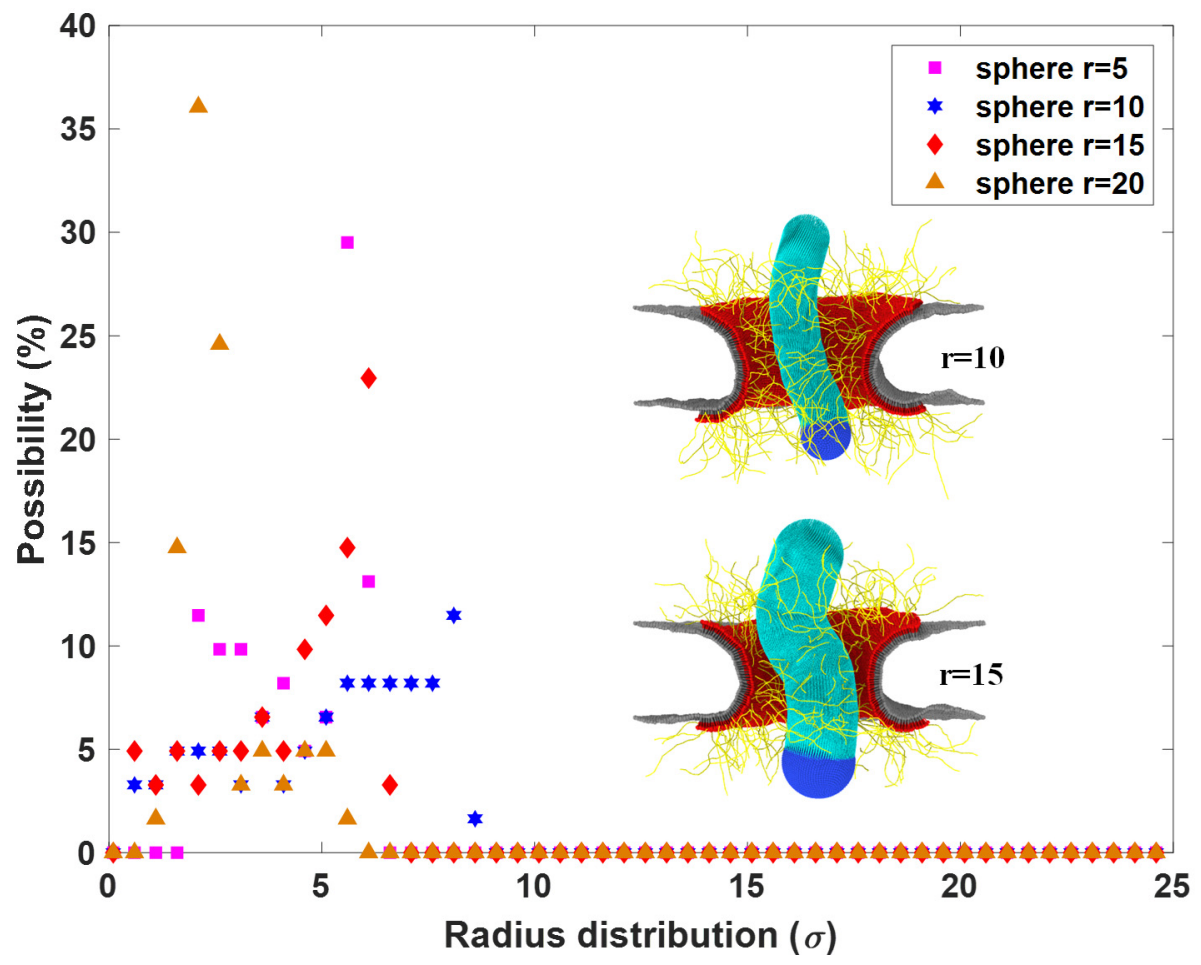

Figure S5. Inert NP distribution inside the NPC channel (top-down view). Radial distance is the distance between the center of the NP and the center axis of the channel.

\section{(4) Effect of shape}

As shown in Figure S7, all the cone-shaped NP is put with different initial orientations with respect to the cone bottom surface plane and $50 \sigma$ height away from the mid-plane of the nucleus. As the simulations goes on, the cone NP experiences the rotation to minimize the local contact energy with the FGN. This phenomenon is similar to the rotation process of cylindrical NP that has been observed and explained in Figure 11 and Figure 12. Although the free energy profile under different orientations follows the same trends as shown in Figure S8, the rotation behavior observed in the snapshots of Figure S7 indicates the non-negligible role of the orientation played in the transport process. 

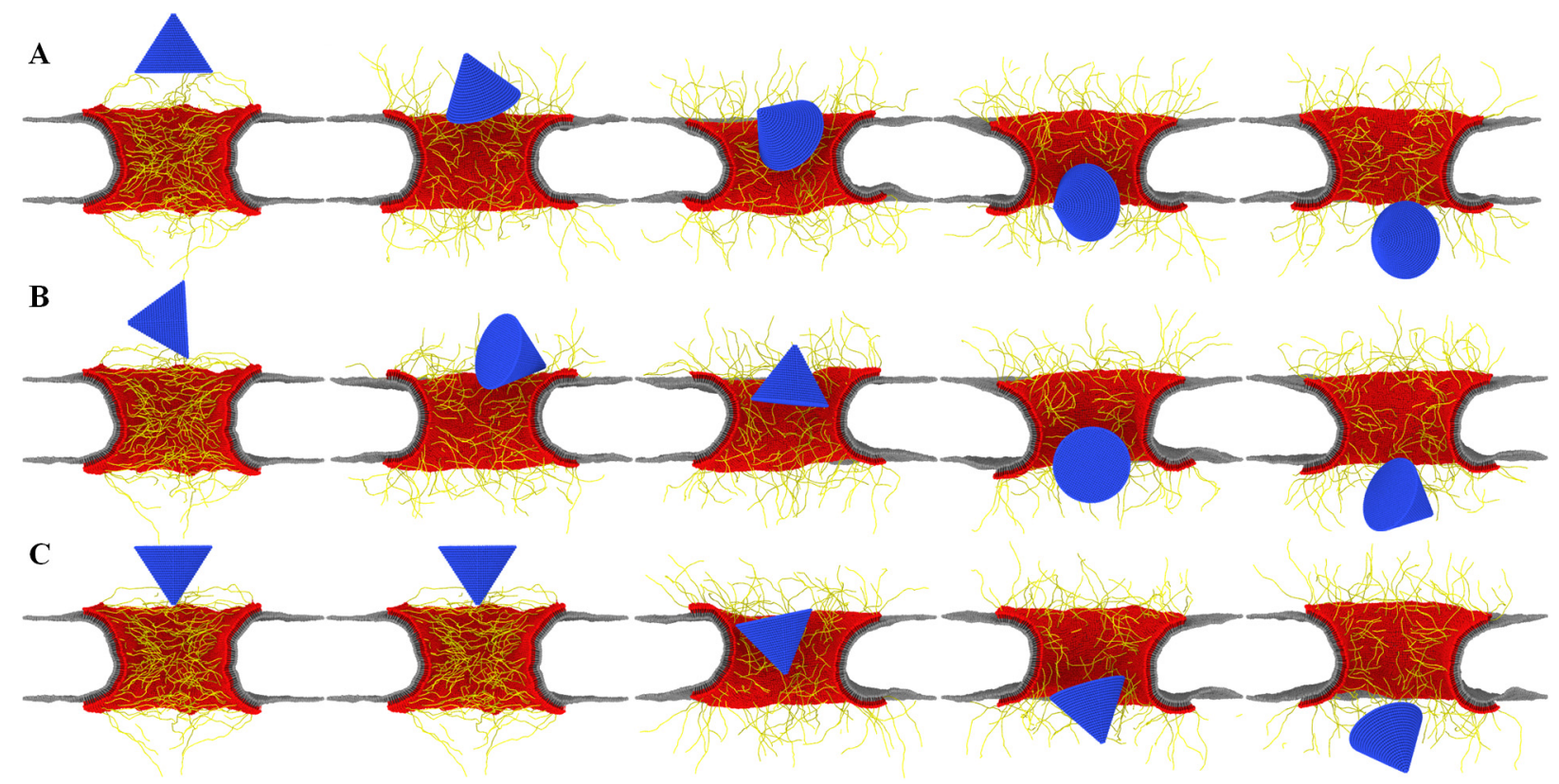

Figure S6. Dynamic cone NP transport process through the NPC channel with different initial orientation.

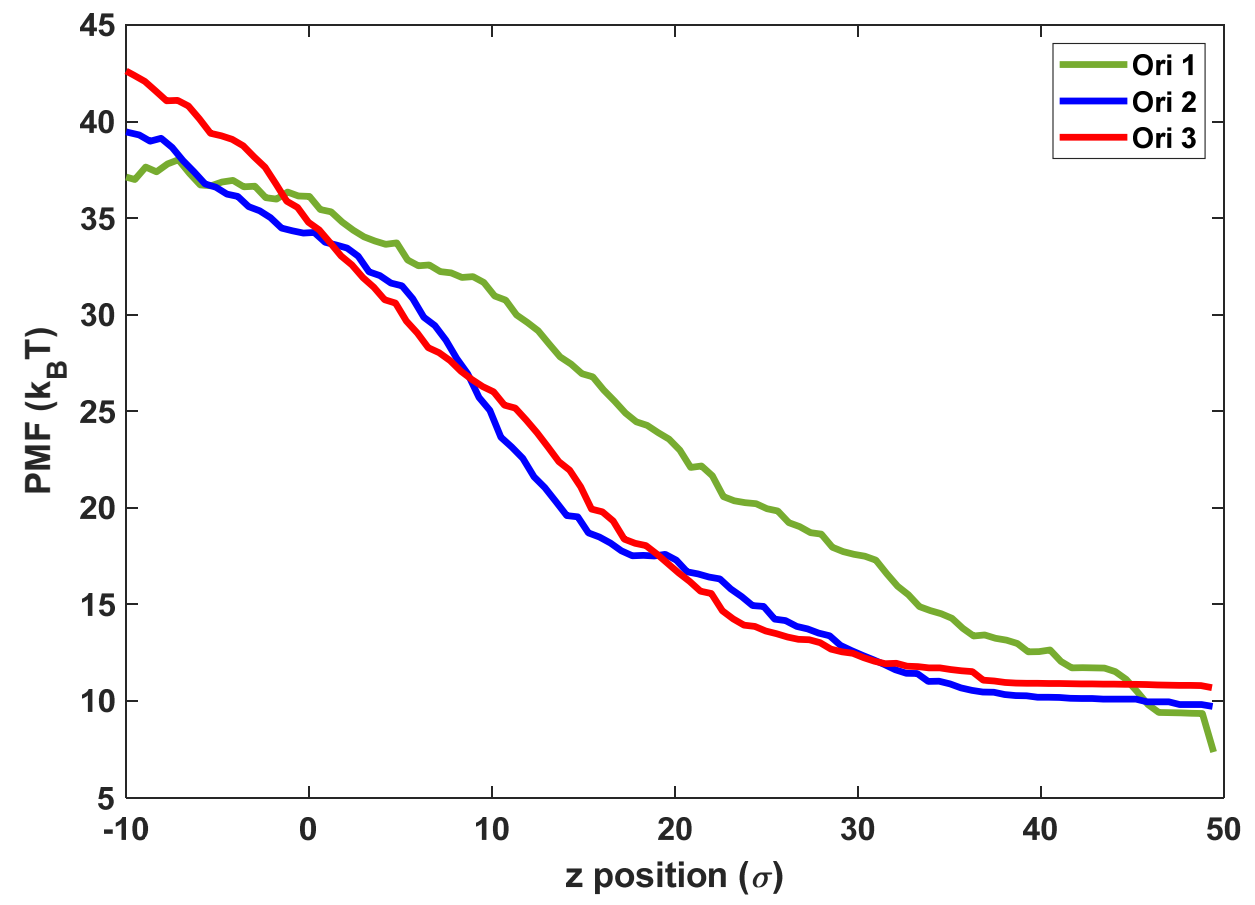

Figure S7. Effect of orientation on the free energy profiles of cone NP's transport process through the NPC channel. 